\title{
Os reassentamentos involuntários em programas de urbanização e de saneamento: os casos Promaben, Prosamim e Probacanga
}

\author{
The involuntary resettlement areas in urbanization and sanitation programs: \\ the cases of Promaben, Prosamim and Probacanga
}

Raifran Abidimar de Castro

Instituto Federal de Educação, Ciência e Tecnologia (IFMA), Açailândia, MA, Brasil

\section{Resumo}

Apresenta-se uma análise sobre os processos de reassentamentos involuntários (RI) implantados em projetos de intervenção urbana. Foram selecionados os programas em implantação nas áreas da Amazônia brasileira: o PROBACANGA, de São Luís, no Maranhão, que recebe financiamento do Banco Internacional para Reconstrução e Desenvolvimento (BIRD); o PROMABEN, de Belém, no Pará, e o PROSAMIM, de Manaus, no Amazonas, dois programas apoiados pelo Banco Interamericano de Desenvolvimento (BID). Realizou-se uma pesquisa bibliográfica e documental, com a utilização de relatórios dos bancos, das prefeituras e dos governos estaduais executores dos programas, complementando-se com textos acadêmicos e com uma base teórica do urbanismo neoliberal. Primeiramente, fez-se uma análise sobre os financiamentos do BIRD e do BID, para, em seguida, mostrar a relação desses dois bancos com o urbanismo neoliberal. Depois, buscou-se uma apresentação da visão de grandes teóricos sobre os processos de RI e de como os bancos citados os operacionalizavam em seus financiamentos. Apresentaram-se as principais características dos três programas, com destaque aos dados e às avaliações sobre os RI. A análise indica que são muitos os indícios de que os reassentamentos utilizados estão relacionados com os preceitos do urbanismo neoliberal. Percebe-se que há uma concorrência entre as cidades e que se prioriza um embelezamento urbano nesses programas. Os RI têm gerado incertezas nas comunidades, além de dificultar o acesso aos serviços públicos básicos.

Palavras-chave: Intervenção urbana. Urbanismo neoliberal. Gentrificação.

\section{Abstract}

We present an analysis of the involuntary resettlement process (IR) deployed in urban development projects. We selected programs being implemented in the Brazilian Amazonia: the "ProBacanga" from São Luis - Maranhão, which receives funding from the IBRD; and two programs supported by the IDB: the "PROMABEN" Belém - Pará; and Manaus - Amazonas, the "PROSAMIM". We conducted a bibliographic and documentary research using reports of banks and state and local governments implementing programs, complemented with academic texts, followed by a theoretical basis of neoliberal urbanism. It begins with an analysis of the IBRD and IDB financing, and their relationship with the neoliberal urbanism, followed by a presentation of major theoretical

RAC é doutorando em Desenvolvimento Socioambiental (NAEA-UFPA); Professor de Geografia do IFMA/Açailândia; Bolsista do PROQUALIS/IFMA, e-mail: raifrancastro@ifma.edu.br 
vision on the IR processes, and how the banks cited the operate their financing. We present the main features of the three programs, with emphasis on IR data and reviews. The analysis shows many indications that the resettlement used in the three programs are related to the principles of neoliberal urbanism. It is noticed that there is a competition among cities, and that these programs prioritize the urban embellishment. The IR has generated uncertainty in communities and prevents access to basic public services.

Keywords: Urban intervention. Neoliberal urbanism. Gentrification.

\section{Introdução}

0 processo de urbanização de diversas capitais brasileiras ocorreu sem que regras rígidas de planejamento fossem aplicadas (Santos, 2005; Maricato, 2001; Carlos, 2001). Para entender o fenômeno urbano, suas diversas variações e seus processos histórico-geográficos nas capitais abrangidas pelo bioma amazônico, Becker (2013) e Ab'Saber (2004) realizaram diversos estudos que confirmaram a tendência nacional nessa região.

Atualmente, destacam-se trabalhos de Trindade (2010), Browder \& Godfrey (2006), Castro (1989, 2008) e Oliveira (2006), nos quais os problemas mais analisados são as ocupações inadequadas e sem planejamento, os precários serviços públicos e os programas de urbanização que se apresentam como uma solução.

Sabe-se que a mobilidade e o saneamento ineficientes, além da precarização dos serviços públicos, são observáveis em diversas cidades amazônicas. $\mathrm{Na}$ tentativa de reduzir esses problemas em capitais como São Luís, no Maranhão, Belém, no Pará, e Manaus, no Amazonas, implantaram-se programas de regularização e de melhoria da qualidade de vida da população, nos quais foram priorizados programas de habitação, de urbanização e de saneamento.

Para que esses programas fossem implantados, realizaram-se os reassentamentos involuntários (RI), que alteraram o cotidiano de grandes parcelas das comunidades (Vainer, 1998). Os RI fazem parte do cotidiano das cidades que realizam grandes projetos de intervenção urbana, principalmente quando é proposto urbanizar áreas classificadas como "precárias".

Mas ainda são poucos os estudos que avaliam os mecanismos e a repercussão dos RI nas populações envolvidas. Com a finalidade de contribuir com esse debate, o objetivo deste artigo é disponibilizar informações sobre processos de RI em programas de urbanização que recebem financiamentos de bancos internacionais ${ }^{1}$, além de complementar com uma análise dos procedimentos utilizados em três capitais abrangidas pelo bioma amazônico brasileiro, pautando-se na abordagem do urbanismo neoliberal.

Foram selecionadas as experiências do PROMABEN em Belém, do PROSAMIM em Manaus e do PROBACANGA em São Luís. Os critérios de seleção foram: realização de ações de RI; ser destaque regional e nacional; disponibilização de documentos e relatórios; aplicação de processos de RI já realizados ou em andamento.

A metodologia aqui aplicada caracteriza-se como uma pesquisa bibliográfica (Lima \& Mioto, 2007) e documental (Sá-Silva et al., 2009). Utilizou-se também dos procedimentos da pesquisa qualitativa (Strauss \& Corbin, 1998; Creswell, 1994), com uso de fontes secundárias (Merriam, 2009; Stewart \& Kamins, 1993), a saber: relatórios disponibilizados pelos sites das prefeituras, nos casos de São Luís e Belém, do site do Governo do Estado do Amazonas e documentos de acompanhamento dos programas e das operações de crédito disponíveis nos sites do Banco Interamericano de Desenvolvimento (BID) e do Banco Internacional para Reconstrução e Desenvolvimento (BIRD), ligado ao Banco Mundial (BM).

Para complementar as análises, foram utilizados estudos que apresentavam caracterizações dos programas, dos órgãos públicos participantes e das populações que foram reassentadas ou que estão previstas para participar desse processo. Essas fontes foram trianguladas (Creswell, 1994) com as citadas anteriormente. Com isso, pôde-se fundamentar as análises com maior acurácia.

\footnotetext{
${ }^{1}$ São tratados o Banco Internacional para Reconstrução e Desenvolvimento (BIRD), que é ligado ao Banco Mundial (BM), e o Banco Interamericano de Desenvolvimento (BID).
} 


\section{O urbanismo neoliberal e os financiamentos do BIRD e do BID}

Críticas à gestão das cidades baseada em objetivos econômicos são apresentadas por diversos pesquisadores, como Acselrad (2009, 2011), Wacquant (2001), Campbell (1996) e Castells (1989), que rejeitam o processo de segregação urbana e também a concentração de famílias pobres em áreas sem condições básicas de sobrevivência. Na tentativa de reduzir essas disparidades, surgem os programas de habitação, de urbanização e de saneamento.

Ao analisar esses programas, Vainer (2003) destaca que, com eles, inicia-se um processo de redução de uma gestão democrática das cidades, para a transformação delas em "cidades empresas" ou "cidades mercadorias", além do incentivo a uma "produção capitalista das cidades", como cita Banerjee-Guha (2008, p. 64).

0 processo de redução dos investimentos nas necessidades básicas dos moradores com a finalidade de priorizar projetos urbanos de interesse de grandes corporações é chamado de urbanismo neoliberal (Smith, 2002). Esse movimento tem ampliado a exclusão social e o deslocamento físico e social de grupos marginalizados (Daher, 2013), assim como a exclusão dos pobres das suas localidades, que é a "gentrificação" (Smith, 2002, p. 446).

A centralização e a fragmentação dos direitos de ação empresarial imobiliária e o processo de "recomposição" das áreas centrais para a realização de grandes projetos, inclusive ligados às "atividades corporativas", são criticados por Farias (2013, p. 14). Já Harvey (1996) complementa ainda que, ao incentivar o "empresariamento" das cidades, se estimula a competição entre elas, o que fortalece o "[...] alinhamento mais forte à disciplina e à lógica do desenvolvimento capitalista" (Harvey, 1996, p. 56). Esses incentivos perpassam por grandes financiamentos.

Como se sabe, os grandes programas de intervenção urbana são, na sua grande maioria, financiados por bancos públicos ou agências internacionais. "Para que o mercado tome suas decisões quanto à produção da cidade neoliberal, ele requer necessariamente recursos públicos e aparatos jurídicos e institucionais que sustentem sua atuação" (Alves \& Rizek, 2014, p. 84). Nesses financiamentos, destacam-se o BIRD (BM) e o BID, que buscam disseminar o ideal das "cidades-modelo" (Sánchez, 2001, p. 31).
Para Melo \& Moura (1990, p. 104 apud Ribeiro, 2013, p. 13), já nas décadas de 1980 e 1990, o BM buscava uma "[...] reestruturação de setores dos aparelhos de Estado, visando à adoção de um formato empresarial nas agências governamentais urbanas, [...] consistente com o credo neoliberal". Já no caso do BID, Santana (2013), Maricato (2009) e Viana \& Fonseca (2006) citam que, por meio dos projetos de reforma urbana, esse banco vem ampliando sua capacidade de instalar uma reforma do Estado.

O contexto anterior amplia a ação empresarial em setores prioritariamente públicos. Como destaca Borges (2015, p. 93), “O BID tem contribuído com a orientação de um modelo de gestão de cidades competitivas e atraentes aos investimentos capitalistas", além de disseminar, nos planejadores locais, os ideais neoliberais aplicados às ações urbanísticas, por meio dos momentos de "capacitação" das equipes executoras dos programas (Maricato, 2009, p. 7). Arantes (2006) e Ribeiro (2005) destacam ainda que os investimentos do BID e BIRD têm ampliado a ação empresarial no seio da administração pública.

Segundo Osmont (2008, p. 67), as políticas de desenvolvimento urbano têm recebido grande apoio dessas organizações financeiras e deixaram o foco social, pois "[...] o objetivo nitidamente afirmado é tornar as cidades ainda mais produtivas". Além disso, para o BM e para o BID, os recursos privados são importantes para a estruturação das cidades (Arantes, 2006, p. 67).

De acordo com Santana (2013, p. 88), todos esses investimentos nos chamados "embelezamentos das cidades" são realizados com a remoção dos moradores com poucos recursos financeiros. Como cita Mayer (2013, p. 299), os reassentamentos reduzem o acesso aos direitos humanos.

Nesse contexto, surge a temática dos RI, que fazem parte do cotidiano das obras de redução das vulnerabilidades e de intervenção urbanística (Pereira, 2011; Zuquim, 2010). Esses programas, que se apresentam como propulsores da melhoria da qualidade de vida dos pobres, além de não se estabelecerem como processos integradores, ampliam as desigualdades de acesso aos bens públicos (Swyngedouw et al., 2002).

Para Hansen \& Oliver-Smith (1982), o RI está entre os principais problemas conflituosos urbanos da atualidade. Os reassentamentos ocorrem por diversos motivos: construção de hidrelétricas (Webber \& McDonald, 2004); induzidos por conflitos (Muggah, 
2000); desastres ambientais (Hutton \& Haque, 2004); mineração (Terminski, 2013); mudanças climáticas/ambientais (Mathur, 2015), além dos impulsionados pelas grandes obras de reestruturação urbana, foco deste artigo.

\section{O reassentamento involuntário para o BIRD e o BID}

Segundo Partridge (1989), o BM foi a primeira agência internacional que elaborou orientações a serem seguidas em processos de reassentamento. Mas existem críticas sobre a sua incapacidade de aplicar suas diretrizes satisfatoriamente (Bartolome, 2000, p. 14).

Os RI impõem uma mudança total de vida (Cernea, 1989, p. 21), pois, na maioria dos casos, não cabe à população o direito de decidir ficar (Wet, 2005). Dentre os problemas relacionados com os RI, encontram-se: os riscos de perda das atividades de subsistência e o empobrecimento (Matos \& Medeiros, 2015; Perera, 2014; Espejel, 2013; Rodrigues, 2013; Bartolome, 2000; Santos, 2007), a dificuldade no planejamento da prevenção destes (Vandergeest, 2003) e a perda das redes de relações dos moradores (Della Noce, 2013).

Para Robinson (2003), resultados positivos podem ser obtidos com os RI quando se aliam reinstalação e reintegração. Já Quetulio-Navarra et al. (2012) acrescentam que o apoio às lideranças comunitárias tende a ampliar a capacidade de restabelecimento das relações comunitárias nas áreas de reassentamento. Resultados positivos também podem ser alcançados se os RI conseguirem representar a possibilidade de que as populações terão acesso às políticas públicas (Oliver-Smith \& Sherbinin, 2014; McDonald et al., 2008).

Mas Batista (2012, p. 39) esclarece que as exigências pelo cumprimento de cronogramas e de agendas rígidas reduzem o potencial de participação das comunidades nas decisões. A pouca ou inexistente participação dos movimentos sociais tem sido uma grande preocupação (Oliver-Smith \& Sherbinin, 2014).

O BIRD e o BID estão entre as instituições que mais financiam projetos de urbanização, de saneamento e de habitação no Brasil. Os investimentos dessas organizações pautam-se, dentre outros focos, no que, segundo eles, é classificado como uma redução da vulnerabilidade social e ambiental de populações desfavorecidas economicamente (Véras, 2010).
O BM (2008, p. 134) cita sua Política Operacional 4.12 (OP 4.12 Involuntary Resettlement) e destaca que os reassentamentos devem ser evitados ou, ao menos, amenizadas as circunstâncias negativas relacionadas a eles. Cita-se ainda que:

[...] se o reassentamento involuntário em projetos de desenvolvimento não for complementado com medidas atenuantes, causa, muitas vezes, graves riscos econômicos, sociais e ambientais: os sistemas de produção são desagregados; pessoas se deparam com um empobrecimento quando perdem o seu patrimônio ou fontes de renda. (BM, 2008, p. 134).

O BID menciona, em sua Política Operacional 710, que "[...] será envidado todo esforço para evitar ou minimizar a necessidade de reassentamento involuntário" (BID, 1998, p. 1), complementando ainda que, caso esse deslocamento seja inevitável, “[...] um plano de reassentamento deve ser elaborado a fim de assegurar que as pessoas afetadas recebam compensação e reabilitação adequadas" (BID, 1998). Tanto o BM quanto o BID condicionam os financiamentos dos programas à aprovação desses planos de reassentamento, como os casos do PROBACANGA, do PROMABEN e do PROSAMIM aqui analisados.

\section{O PROBACANGA}

Um exemplo que bem representa a questão dos RI em áreas urbanas da Amazônia é o caso de São Luís, com o Programa de Recuperação Ambiental e Melhoria da Qualidade de Vida da Bacia do Bacanga (PROBACANGA), que prevê um plano de reassentamento involuntário (PRI) da margem esquerda do rio. Trata-se da maior bacia hidrográfica na área da Ilha do Maranhão, com 10.475,61 hectares (Oliveira, 2008, p. 28). Além disso, é a segunda em concentração urbana da capital maranhense (Nascimento, 2010, p. 26), ocupação que ocorreu de forma irregular (Martins, 2008).

Nesta bacia, o processo de ocupação exerce grande pressão sobre os recursos hídricos, inclusive nas áreas de nascentes (Vinhote, 2008). Silva et al. $(2006,2015)$ destacam que os lançamentos de resíduos, a poluição industrial e a retirada da vegetação ciliar têm causado sérios danos socioambientais - características que situaram a Bacia do Bacanga dentre as prioridades de intervenção, inclusive com a realização de RI. 
Destaca-se que as obras relacionadas com esse RI fazem parte do Acordo de Empréstimo (AE) no 7578-BR, firmado entre a Prefeitura Municipal de São Luís (PMSL) e o BIRD, ainda no ano de 2008 (BIRD, 2008). A previsão para encerramento do projeto era dezembro de 2013, mas recentemente recebeu um aditivo de 22 meses por conta dos atrasos nas obras.

Sobre os reassentamentos, citam-se os casos dos bairros Sá Viana e Vila Coroadinho, que, em diversos anos, já passaram por inundações (São Luís, 2006). Mesmo com esse risco, os moradores dessas áreas chegaram a fazer manifestações contra esse RI, até mesmo com a interrupção do trânsito sobre a Barragem do Bacanga. Dentre as reinvindicações, destacam-se: a maior disponibilização de informações sobre o RI da área e a avaliação da possibilidade de permanência no local.

Depois dessa manifestação, algumas reuniões foram realizadas, nas quais a Secretaria Municipal de Projetos Especiais (SEMPE) buscou explicar as condições do reassentamento. Também foi "esclarecido" que a mudança para o "Residencial Piancó" ${ }^{2}$ não era obrigatória, mas opcional (0 4oPoder, 2014). Contudo, o fato é que a área deveria ser desocupada para as obras.

A previsão era destinar R \$7.672.000,00 para o PRI, com a construção de 500 casas - valor este relacionado aos $\mathrm{R} \$ 59.400 .000,00$ que seriam investidos em todas as ações do programa. Desse total, $\mathrm{R} \$ 35.640 .000,00$ foram provenientes de financiamento do BIRD, e $\mathrm{R} \$ 23.760 .000,00$, de contrapartida da PMSL (São Luís, 2007).

A primeira etapa do reassentamento do PROBACANGA ocorreu ainda no ano de 2014. Foram entregues 108 apartamentos para famílias provenientes dos bairros Sá Viana, Jambeiro e Piancó (SEMPE, 2015c). Segundo o secretário municipal de projetos especiais, Gustavo Marques (2015 apud SEMPE, 2015d), “[...] os apartamentos do Residencial Piancó foram uma das alternativas encontradas pelo Programa Bacia do Bacanga para reassentar esses moradores". Segundo o BM (BM, 2015, p. 9), a satisfação das famílias estava em um nível aceitável.

Sobre as outras etapas do RI do PROBACANGA, a especialista socioambiental da SEMPE, Eloina Reis, destaca que:

\footnotetext{
2 Este residencial faz parte das obras do "Minha Casa, Minha Vida" do Governo Federal. Não tem relação direta com o programa, mas tem sido utilizado para facilitar o reassentamento das famílias.
}

Os moradores que viviam em áreas que passaram por intervenções com a obra do canal receberão novas casas, próximas as suas antigas residências, podendo assim manter os vínculos sociais já estabelecidos. (SEMPE, 2015a).

Segundo a SEMPE (2015b), no mês de abril de 2015 , foram realizados os cadastros para o que seria um reassentamento "voluntário" das famílias residentes em áreas de risco, nos bairros Sá Viana, Jambeiro e Piancó.

A etapa citada se trata do reassentamento de famílias que atenderam às solicitações da prefeitura e pretendiam sair das suas atuais moradias sem a necessidade de que os executores do projeto recorressem às vias judiciais. Segundo a secretaria (SEMPE, 2015b), os moradores que se enquadrassem nos requisitos do programa "Minha Casa, Minha Vida" participariam do processo de reassentamento para o Residencial Piancó. Mas não foram discriminadas as iniciativas que seriam tomadas com os que, por ventura, não preenchessem os requisitos.

Em relatório divulgado em abril de 2015, o BM (2015) destaca que essas 108 famílias representavam apenas $18 \%$ de um total de 595 que deveriam passar pelo processo de reassentamento. No documento, cita-se ainda que o "[...] reassentamento das famílias que vivem em áreas de risco na margem esquerda (Sá Viana) se move mais devagar" (BM, 2015, p. 2, tradução nossa). Essa demora traz problemas aos moradores, pois eles não têm como planejar suas vidas.

O canal do Rio das Bicas, que é componente do PROBACANGA, também vem passando por processos de reassentamento, com destaque para as obras de nivelamento do solo no bairro Salinas do Sacavém, onde seriam construídas 12 casas para moradores de áreas de risco (SEMPE, 2015e). Além disso, foram reassentadas da área para a realização das obras um total de 30 famílias, "[...] que residiam em uma área de influência das obras do canal. Com a indenização feita pela prefeitura foi possível o reassentamento dos moradores" (SEMPE, 2015f).

Como se percebe, dois fatores são complicadores aos cotidianos dos moradores: os atrasos nas obras e a falta de informações precisas sobre os RI a que devem ser submetidos. Os RI não têm sido realizados de forma conjunta, mas vêm solucionando problemas esporádicos com pequenos grupos de moradores, fragmentando comunidades em diversas áreas. 


\section{O PROMABEN}

Para Pereira (2009), a grande quantidade de localidades com ocupação irregular em Belém tem relação direta com os baixos investimentos no fortalecimento e na regularização de áreas ocupadas pela população de baixa renda. Para amenizar esse problema histórico, foram iniciados os trabalhos de regularização fundiária, de saneamento e de urbanização de diversos locais problemáticos.

Dentre as iniciativas, a de maior destaque é o Programa de Saneamento da Bacia da Estrada Nova (PROMABEN), que se encontra na sua segunda etapa, denominada de PROMABEN II. Na Tabela 1, há uma descrição das principais informações de cada fase. Segundo a Prefeitura Municipal de Belém (PMB), o restante dos recursos que não são financiados pelo BID provém da própria prefeitura (Belém, 2014a, b).

Nos documentos oficiais, como o Relatório de Impactos ao Meio Ambiente (RIMA) do PROMABEN (Belém, 2007, p. 79-80), o objetivo do reassentamento "[...] é liberar estas áreas para implantar tais obras considerando o resgate da cidadania como condição para a sustentabilidade do PROMABEN" (Belém, 2007, p. 80). De acordo com o Relatório de Término de Projeto (BID, 2013), no item de Reordenamento Urbano e Reassentamento de Famílias e de Negócios, tratando-se especificamente de investimentos, foram implantadas "Soluções residenciais para aproximadamente 1.037 famílias e cerca de 450 residências ou pequenos negócios" (BID, 2013, p. 3), não explicitadas.

Para as ações de reassentamento, havia uma previsão de gastos de 3\% (Santana, 2012). Dessa forma, segundo esse autor, o programa “[...] deverá reproduzir a tendência de impermanência de famílias após o reassentamento [...]" (Santana, 2012, p. 27).
Já para os que ficam, disseminam-se os discursos da satisfação em permanecer em um local urbanisticamente mais aparelhado (Cardoso, 2014, p. 69).

Como nos apresenta Pereira (2009), todo o processo de construção do complexo de obras resulta em uma valorização imobiliária. Inicia-se, assim, o processo de especulação pelas grandes empresas. Por exemplo, está em andamento a construção de condomínios cujos apartamentos mais valorizados podem chegar a $\mathrm{R} \$ 1.200 .000,00$ (Pereira, 2009, p. 69). Enquanto isso, persistem as defesas dos reassentamentos.

Para Sousa et al. (2014, p. 33), "[...] os impactos socioeconômicos na vida das famílias que foram removidas demonstraram uma série de perdas como acesso ao trabalho, diminuição de renda e perda nas relações de vizinhança". Cruz et al. (2015, p. 242) salientam ainda que isso resulta em uma desarticulação de "[...] relações historicamente construídas, com as quais estão relacionadas diretamente às questões da moradia e do trabalho", além das significativas alterações nas feiras e nos pequenos portos, que são utilizados para escoamento e comercialização das produções dos moradores da área (Araújo, 2013, p. 194).

Para dar continuidade e também para atender aos passivos ambientais da fase I do programa, está em início o PROMABEN II. De acordo com a Operação de Crédito BR-L1369, serão realizadas intervenções similares às da primeira fase na área do bairro Jurunas, do conjunto Aluisio Chaves e do canal da Quintino Bocaiuva. Nessas localidades, se prevê a construção de habitações para o reassentamento (BID, 2014, p. 3). No entanto, informações precisas sobre esses RI e sobre a avaliação pelos moradores não são disponibilizadas.

Tabela 1 - Valores, áreas de abrangência e quantidade prevista de famílias para reassentamento involuntário (RI) em cada etapa do PROMABEN

\begin{tabular}{lcclc}
\hline \multicolumn{1}{c}{ Programa } & Início & $\begin{array}{c}\text { Valores } \\
\text { (US\$) }\end{array}$ & \multicolumn{1}{c}{ Localidade abrangida } & $\begin{array}{c}\text { Famílias } \\
\text { reassentadas }\end{array}$ \\
\hline $\begin{array}{l}\text { PROMABEN I } \\
(1998 / \text { OC-BR })^{\star}\end{array}$ & 2008 & 145,8 milhões $^{\star \star}$ & $\begin{array}{l}\text { Canais Timbiras, Quintino Bocaiuva, 3 de Maio, Bernardo Sayão, Caripunas, } \\
\text { Doutor Moraes e 14 de Março }\end{array}$ & 1.100 \\
\hline $\begin{array}{l}\text { PROMABEN II } \\
(3303 / \text { OC-BR })^{\star \star}\end{array}$ & 2015 & 250 milhões $^{\star \star \star}$ & $\begin{array}{l}\text { Canais Caripunas e Bernardo Sayão; Orla do Rio Guamá (ambas consideran- } \\
\text { do a área central do bairro Jurunas) }\end{array}$ & 410 \\
\hline
\end{tabular}

*0perações de crédito de cada etapa do programa. **Financiamento de US\$ 68,7 milhões pelo BID. ${ }^{\star \star \star F i n a n c i a m e n t o ~ d e ~ U S \$ ~} 125$ milhões pelo BID.

Fonte: Elaborada pelo autor com dados de BID $(2013,2014)$. 


\section{O PROSAMIM}

O PROSAMIM foi criado no ano de 2003, apresentado com a função de modernizar e de estruturar a cidade de Manaus, considerando os conceitos urbanísticos atuais e de beleza paisagística construída. Para Freitas et al. (2010), o programa trouxe benefícios sociais e ambientais para as comunidades do município.

Na época, propagava-se a intenção de reassentar em torno de 7 mil famílias para locais considerados com melhor estrutura urbana na etapa PROSAMIM I (Amazonas, 2012a). Ao término da primeira fase, foram remanejadas 6.683 famílias (Amazonas, 2012a). No caso da fase II, “[...] na área do Igarapé do Quarenta e afluentes, o total previsto para reassentamento é de 4.543 famílias que vivem em casas, anualmente afetadas por inundações. Até janeiro de 2014 já foram reassentadas 2.887 famílias" (Amazonas, 2014).

Segundos os dados do plano de reassentamento da etapa III (Amazonas, 2012b), nas Bacias dos Igarapés de Manaus, Bittencourt, Mestre Chico, Quarenta, Cachoeirinha, Franco, Treze de Maio, Sapolândia, Bombeamento, Franceses e Passarinho, havia uma estimativa de que 8.388 famílias fossem reassentadas. No caso da Bacia do Igarapé São Raimundo, seriam afetadas 3.451 famílias, ou seja, 95\% estariam em área de risco à inundação (Amazonas, 2012b).

A etapa III está em fase de implantação, mas já foram reassentadas 2.887 famílias. No entanto, ainda faltam outras 1.656, originárias dos bairros Japiim II e Cachoeirinha (Amazonas, 2014). Vale destacar que, em todas as fases, o PROSAMIM acarretou no reassentamento de $77,26 \%$ dos moradores para bacias hidrográficas urbanas de Manaus (Batista, 2013, p. 271).
Na Tabela 2, são apresentados os valores, as localidades de abrangência e a quantidade de famílias a serem reassentadas. Os valores que excedem os financiamentos do BID se referem aos investimentos do próprio Governo do Estado do Amazonas e deste em parceria com a Caixa Econômica Federal, além do Programa de Aceleração do Crescimento (PAC), do Governo Federal.

Algo preocupante sobre o planejamento desses RI no âmbito do PROSAMIM é relatado pela equipe de avaliação do próprio BID, quando cita que: "[...] o ritmo do remanejamento pode vir a sofrer um processo de desaceleração devido à redução de oferta de unidades habitacionais no mercado imobiliário popular [...]" (Amazonas, 2012a, p. 16). Além disso, menciona que apenas 35\% dos moradores que optaram pelo bônus moradia, que era no valor de $\mathrm{R} \$ 21.000,00$, conseguiram encontrar moradias na mesma área de origem e que $30 \%$ perderam suas rendas, pois suas atividades eram desenvolvidas nas próprias moradias (Amazonas, 2012a, p. 17).

Existem sérios indícios de redução quantitativa de moradores em algumas áreas em que foi implantado o PROSAMIM, como nos apresentam Cruz \& Sá (2012), com destaque para os bairros localizados na Bacia do Educando. Nessa localidade "[...] passaram de 308.340 habitantes no ano 2000 para 286.488 habitantes em 2010, com uma diferença de 21.852 (7\%) pessoas que deixaram as áreas" (Cruz \& Sá, 2012, p. 14). Há, portanto, a constatação de que esses RI estão diretamente relacionados com a perda de qualidade de vida das populações que são submetidas a esses programas.

Tabela 2 - Valores, áreas de abrangência e quantidade prevista de famílias para reassentamento involuntário (RI) em cada etapa do PROSAMIM

\begin{tabular}{lcccc}
\hline \multicolumn{1}{c}{ Programa } & Início & Valores (US\$) & Localidade abrangida & $\begin{array}{c}\text { Famílias } \\
\text { reassentadas }\end{array}$ \\
\hline $\begin{array}{l}\text { PROSAMIM I } \\
(1692 / O C-B R)^{*}\end{array}$ & 2006 & 200 milhões & Bacia dos Igarapés Educandos e Quarenta & 5.661 \\
\hline $\begin{array}{l}\text { PROSAMIM II } \\
(2006 / 0 C-B R)^{*}\end{array}$ & 2008 & 220 milhões $^{\star * *}$ & Bacia do lgarapé São Raimundo & 4.352 \\
\hline $\begin{array}{l}\text { PROSAMIM III } \\
(2676 / O C-B R)^{*}\end{array}$ & 2012 & 400 milhões $^{\star \star \star *}$ & Bacia do lgarapé São Raimundo & 4.750 \\
\hline
\end{tabular}

${ }^{\star}$ Operaç̃̃es de crédito de cada etapa do programa. ${ }^{\star \star}$ Financiamento de US\$ 140 milhões do BID. ${ }^{\star \star \star}$ Financiamento de US\$ 154 milhões do BID. ${ }^{\star \star \star \star * i n a n c i a m e n t o ~ d e ~ U S \$ ~}$ 280 milhões do BID.

Fonte: Elaborada pelo autor com dados do site do BID (2015). 


\section{Considerações finais}

Uma análise sobre os RI nos programas de intervenção urbana aqui apresentados, a partir do urbanismo neoliberal, torna-se complicada quando não há uma grande quantidade de informações disponíveis. Mas a base teórica, os processos de implantação dos programas e a correlação desses RI com os objetivos gerais dos programas abrem espaço para essa possibilidade.

Para isso, algumas perguntas devem ser respondidas: 1) Há uma disputa entre as cidades citadas a fim de receberem mais investimentos? 2) Esses programas visam melhorar a qualidade de vida das populações pobres ou visam a um "embelezamento" urbano? 3) Observam-se processos de deslocamento físico e social dos grupos marginalizados? 4) Existem mecanismos que ampliam uma lógica capitalista de gestão urbana?

Sobre a disputa, deve-se citar que as três cidades sempre pleiteiam o título de "Portal da Amazônia". Além disso, a disputa pela "sede amazônica" da Copa do Mundo de 2014 evidenciou o quanto as três cidades se autointitulam capitais amazônicas. As questões da mobilidade, da segurança e do atrativo turístico pesaram na decisão por Manaus. Esses são apenas dois exemplos. Há ainda as disputas por investimentos em transportes e em plantas industriais. Os programas de intervenção urbana aqui citados são amplamente propalados como investimentos sociais na melhoria da qualidade de vida da população, visando disseminar ideias de cidades suscetíveis às mudanças que investem em grandes ações sociais e de adequação de áreas precariamente ocupadas.

Para reduzir essa precarização urbana, os programas não objetivam apenas o saneamento, mas utilizam-se de reassentamentos para alterar completamente a estrutura da área afetada. Como exemplo, tem-se o PROMABEN, que vem transformando áreas residenciais em estruturas para atrativos turísticos - o que responde à segunda pergunta.

Há um extremo processo de alteração do cotidiano das famílias que passam pelos RI, como se observou nos casos do PROBACANGA e do PROSAMIM. Além disso, são grandes as incertezas de quando e para onde serão reassentadas. Na maioria dos casos, os moradores são transferidos de casas para apartamentos, geralmente com áreas menores, distantes dos seus locais de origem e dos serviços públicos básicos.
Com a somatória das análises citadas, os processos de reassentamento implantados nos três programas avaliados demonstram claras tendências de urbanismo neoliberal. Dessa forma, percebe-se que, ao apoiar financeiramente instituições que realizam esses RI, o BIRD e o BID também apoiam essa tendência e a incentivam. Há, portanto, três exemplos de atuação da lógica capitalista na gestão urbana e, principalmente, no relacionamento com comunidades pobres.

Entretanto, deve-se observar o fato de que esses programas trazem ganhos urbanos na medida em que melhoram o saneamento nas áreas afetadas. Existem casos em que as famílias aprovam os programas e seus respectivos RI, os quais têm seu andamento acompanhado pelo BIRD e BID. Mas não se pode deixar de destacar que os problemas citados pelos pesquisadores e pelas famílias reassentadas ocorrem reiteradamente.

Considerando o exposto, espera-se que este artigo venha contribuir para o debate sobre a execução e a avaliação dos RI em grandes programas de intervenção urbana, que haja uma maior discussão sobre os mecanismos de intervenção das comunidades a serem reassentadas durante todo o processo e que se busque, verdadeiramente, a melhoria da qualidade de vida dessa parcela da população, e não apenas a melhoria da propaganda política, econômica e, sobretudo, comercial das cidades.

\section{Referências}

Ab'Saber, A. N. (2004). Amazônia: do discurso à práxis. São Paulo: USP.

Acselrad, H. (2011). Desregulamentação, contradições espaciais e sustentabilidade urbana. Revista Paranaense de Desenvolvimento-RPD, 107, 25-38.

Acselrad, H. (Ed.). (2009). A duração das cidades: sustentabilidade e risco nas políticas urbanas. Rio de Janeiro: Lamparina.

Alves, M. R., \& Rizek, C. S. (2014). Cidade Contemporânea, Cidade do Empresariamento: aspectos da produção socioespacial do urbano. In J. E. B. Rodrigues, M. R. C. F. Rufino, V. M. Arango, \& D. T. Santos (Eds.), Os estudos socioespaciais: cidades, fronteiras e mobilidade humana (pp. 80-93). Manaus: EDUFAM. 
Amazonas. Governo do Estado do Amazonas. (2012a). Prosamim I. Manaus. Recuperado em 16 de novembro de 2015, de http://prosamim.am.gov.br/o-prosamim/ prosamim-i/

Amazonas. Governo do Estado do Amazonas. (2012b). Programa social e ambiental dos Igarapés de Manaus: plano específico de desapropriação, reassentamento e locação. Manaus. Recuperado em 17 de novembro de 2014, de http://prosamim.am.gov.br/wp-content/uploads/2012/04/ plano-reassentamento-prosamim3.pdf

Amazonas. Governo do Estado do Amazonas. (2014). Prosamim II: obras e reassentamento. Manaus. Recuperado em 16 de novembro de 2014, de http://prosamim.am.gov. br/comunicacao/sala-de-imprensa/videos/

Arantes, P. F. (2006). 0 ajuste urbano: as políticas do Banco Mundial e do BID para as cidades. Revista do Programa de Pós-Graduação em Arquitetura e Urbanismo da FAU-USP, 20(20), 60-75. http://dx.doi.org/10.11606/issn.23172762.v0i20p60-75.

Araújo, A. C. R., Jr. (2013). Apropriação e usos do litoral urbano de Belém-Pará: Portal da Amazônia em questão. Revista do Departamento de Geografia, 25(39), 183-199.

Banco Interamericano de Desenvolvimento - BID. (1998). Operational Policy OP-710. Washington. Recuperado em 14 de novembro de 2014, de http://idbdocs.iadb.org/ wsdocs/getdocument.aspx?docnum=2032100

Banco Interamericano de Desenvolvimento - BID. (2013). Programa de Saneamento da Bacia da Estrada Nova PROMABEN. Projeto BR-L1065. Washington. Relatório de Término de Projeto. Recuperado em 12 de novembro de 2014, de http://idbdocs.iadb.org/wsdocs /getdocument. aspx?docnum $=38196159$

Banco Interamericano de Desenvolvimento - BID. (2014). Programa de Saneamiento Básico de la Cuenca Estrada Nova II (PROMABEN II), Número del Proyecto: BR-L1369. Washington. Recuperado em 12 de novembro de 2014, de http://idbdocs.iadb.org/wsdocs/getdocument. aspx?docnum $=37881616$

Banco Interamericano de Desenvolvimento - BID. (2015). Programa Social y Ambiental para los Igarapés de Manaus (PROSAMIM I, II E III). Washington. Recuperado em 10 de fevereiro de 2015, de http://www.iadb.org/es/proyectos/ project-details,1301.html?Country $=\&$ Sector $=\&$ Status $=\& q$ uery=prosamim
Banco Internacional de Reconstrução e Desenvolvimento - BIRD. (2008). Acordo de Empréstimo No 7578-BR firmado com a Prefeitura Municipal de São Luís. Brasília. Recuperado em 14 de fevereiro de 2015, de http://www.worldbank.org/ projects/P094315/br-municipal-apl-sao-luis-enhancingmunicipal-governance-quality-life-project?lang=en

Banco Mundial - BM. (2008). Licenciamento Ambiental de Empreendimentos Hidrelétricos no Brasil: uma contribuição para o debate (em três volumes). Volume III: Anexos Técnicos - Política Operacional 4.12. Brasília: Banco Mundial no Brasil.

Banco Mundial - BM. (2015). BR Municipal APL: Sao Luis Enhancing Municipal Governance and Quality of Life Project (P094315). Brasília: Banco Mundial no Brasil. Recuperado em 20 de setembro de 2015, de http://www-wds.worldbank. org/external/default/WDSContentServer/WDSP/LCR/ 2015/04/01/090224b082d26f89/1_0/Rendered/PDF/ Brazil000BR0Mu0Report000Sequence012.pdf

Banerjee-Guha, S. (2008). Space relations of capital and new economic enclaves: SEZs in India'. Economic and Political Weekly, 43(47), 51-59.

Bartolome, L. J. (2000). Displacement, resettlement, rehabilitation, reparation, and development. Cape Town: World Commission on Dams.

Batista, S. P. M. (2012). 0 adensamento urbano consolidado em igarapés, como proposta para o desenvolvimento local: o caso do PROSAMIM em Manaus. GEOUSP: Espaço e Teтpo, 31(31), 33-43. http://dx.doi.org/10.11606/ issn.2179-0892.geousp.2012.74267.

Batista, S. P. M. (2013). Injustiça ambiental: o caso do PROSAMIM (Tese de doutorado em Geografia). Universidade de São Paulo, São Paulo.

Becker, B. (2013). A urbe amazônica: a floresta e a cidade. Rio de Janeiro: Garamond.

Belém. Prefeitura Municipal. (2007). EIRA/RIMA PROMABEM - TOMO I. Belém. Recuperado em 15 de novembro de 2015, de http://www.belem.pa.gov.br/promaben /documentos/ Promaben_I/EIA-RIMA-ROMABEN/RIMA/TOMO_I/SA_PR1 09_06_TX_26_005_A.pdf

Belém. Prefeitura Municipal de Belém. (2014a). O projeto Vila da Barca. Belém. Recuperado em 12 de novembro de 2014, de http://www.belem.pa.gov.br/app/c2ms/v/?id=9 \&conteudo $=2745$

Belém. Prefeitura Municipal de Belém. (2014b). Síntese do PROMABEN II: programa de saneamento básico da Bacia da 
Estrada Nova II. Belém. Recuperado em 12 de novembro de 2014, de http://www.belem.pa.gov.br/promaben/ documentos/Promaben_II/Sintes e_Promaben_II_Set2014.pdf

Borges, V. F. (2015). Reflexões sobre a dimensão política do trabalho social em projetos de urbanização. Sociedade em Debate, 21(2), 84-99.

Browder, J., \& Godfrey, B. (2006). Cidades da floresta: urbanização, desenvolvimento e globalização na Amazônia Brasileira. Manaus: UFAM.

Campbell, S. (1996). Green cities, growing cities, just cities?: Urban planning and the contradictions of sustainable development. Journal of the American Planning Association, 62(3), 296-312. http://dx.doi.org/10.1080/01944369608975696.

Cardoso, D. L. (2014). A função estratégica do assistencialismo na produção do espaço na metrópole (Dissertação de mestrado em Geografia Humana). Universidade de São Paulo, São Paulo.

Carlos, A. F. A. (2001). Espaço-tempo na metrópole: a fragmentação da vida cotidiana. São Paulo: Contexto.

Castells, M. (1989). The informational city: information technology, economic restructuring, and the urban-regional process. Cambridge: B. Blackwell.

Castro, E. M. R. (1989). A questão urbana na Amazônia. In: Pará. Secretaria de Estado de Educação. Estudos e problemas amazônicos: história social e econômica e temas especiais (pp. 165-176). Belém: IDESP.

Castro, E. M. R. (2008). Urbanização, pluralidade e singularidades das cidades amazônicas. In E. M. R. Castro (Ed.), Cidades na floresta (pp. 13-39). São Paulo: Anablume.

Cernea, M. M. (1989). Involuntary Resenttlement in development projects. Washington: BID.

Creswell, J. W. (1994). Research design: qualitative, quantitative, and mixed methods approaches. Thousand Oaks: Sage.

Cruz, S. H. R., \& Sá, M. E. R. (2012). Grandes projetos urbanos e expansão da fronteira na Amazônia: portal da Amazônia, em Belém/PA e PROSAMIM, em Manaus/AM. In Anais do III Encontro da SBSNorte. Manaus: UFAM.

Cruz, S. H. R., Silva, I. S., \& Sá, M. E. R. (2015). Segregação social do trabalho e da moradia: "Portal da Amazônia" em Belém (PA). Temporalis, 15(29), 223-246.

Daher, R. F. (2013). Neoliberal urban transformations in the arab city: meta-narratives, urban disparities and the emergence of consumerist utopias and geographies of inequalities in Amman. Environnement Urbain: Urban Environment, 7, 99-115. http://dx.doi.org/10.7202/1027729ar.

Della Noce, L. G. (2013). 0 reassentamento como fator de transformação urbana: o papel do capital social e das redes sociais. In Anais do XV Encontro Nacional da ANPUR. Recife: ANPUR.

Espejel, A. G. (2013). Impacto social de proyectos hidráulicos: una aproximación a una política del reacomodo social en México. Vegueta: Anuario de la Facultad de Geografía e Historia, 13, 77-94.

Farias, J. A., Fo. (2013). A prática urbanística no nacional desenvolvimentismo: velhos ensinamentos para o presente em andamento. In Anais do XV Encontro Nacional da ANPUR. Recife: ANPUR.

Freitas, K. A. A., Barbosa, J., Fo., Pio, N. S., Silva, F. F., \& Moraes, L. S. (2010). Valoração econômica dos benefícios ambientais percebidos pela população da bacia do Educandos provenientes do PROSAMIM. Acta Amazonica, 40(3), 509-514. http://dx.doi.org/10.1590/S0044-59672010000300009.

Hansen, A., \& Oliver-Smith, A. (1982). Involuntary migration and resettlement: the problems and responses of dislocated people. Boulder: Westview.

Harvey, D. (1996). Do gerenciamento ao empresariamento: a transformação da administração urbana no capitalismo tardio. Espaço \& Debates, 16(39), 48-64.

Hutton, D., \& Haque, C. E. (2004). Human vulnerability, dislocation and resettlement: adaptation processes of river-bank erosion-induced displacees in Bangladesh. Disasters, 28(1), 41-62. http://dx.doi.org/10.1111/j.03613666.2004.00242.x. PMid:15016105.

Lima, T. C. S., \& Mioto, R. C. T. (2007). Procedimentos metodológicos na construção do conhecimento científico: a pesquisa bibliográfica. Revista Katálysis, 10(1), 37-45.

Maricato, E. (2001). Brasil, cidades: alternativas para a crise urbana. São Paulo: Vozes.

Maricato, E. (2009). Globalização e política urbana na periferia do capitalismo. VeraCidade, 6(4), 1-25.

Martins, A. L. P. (2008). Avaliação da qualidade ambiental da bacia hidrográfica do Bacanga (São Luis - MA), com base nas variáveis físico-químicas, biológicas e populacionais: subsídios para um manejo sustentável (Dissertação de mestrado em Sustentabilidade de Ecossistemas). Universidade Federal do Maranhão, São Luís. 
Mathur, H. M. (2015). Climate change and displacement: learning from resettlement in the development context. Social Change, 45(1), 118-130. http://dx.doi. org/10.1177/0049085714561939.

Matos, E. A. C., \& Medeiros, R. M. V. (2015). Acumulação por espoliação: uma reflexão sobre a sua introdução em Moçambique. Revista IDeAS, 7, 228-259.

Mayer, B. (2013). Development is no excuse for human rights abuses: framing the responsibility of international development agencies. Trade, Law and Development, 5(2), 286-343.

McDonald, B., Webber, M., \& Yuefang, D. (2008). Involuntary resettlement as an opportunity for development: the case of urban resettlers of the Three Gorges Project, China. Journal of Refugee Studies, 21(1), 82-102. http://dx.doi. org/10.1093/jrs/fem052.

Merriam, S. B. (2009). Qualitative research: a guide to design and implementation. San Francisco: Jossey-Bass.

Muggah, R. (2000). Through the Developmentalist's Looking Glass: Conflict-Induced Displacement and Involuntary Resettlement in Colombia. Journal of Refugee Studies, 13(2), 133-164. http://dx.doi.org/10.1093/jrs/13.2.133.

Nascimento, J. D. (2010). O índice de sustentabilidade ambiental do uso da água como ferramenta de contribuição às políticas públicas de desenvolvimento e conservação na bacia do rio Bacanga, São Luis, MA (Dissertação de mestrado em Sustentabilidade de Ecossistemas). Universidade Federal do Maranhão, São Luís.

0 4o Poder. (2014). Prefeitura discute reassentamento de famílias para o Piancó durante nova reunião. São Luís. Recuperado em 15 de julho de 2015, de http://www. oquartopoder.com/2014/ 02/11/prefeitura-discutereassentamento-de-familias-para-o-pianco-durante-novareuniao/

Oliveira, C. C. C. (2008). Ocupação urbana na bacia hidrográfica e sua influência nas formações ciliares do Rio Bacanga (Trabalho de conclusão de curso em Ciências Aquáticas). Universidade Federal do Maranhão, São Luís.

Oliveira, J. A. (2006). A cultura, as cidades e os rios na Amazônia. Ciência e Cultura, 58(3), 27-29.

Oliver-Smith, A., \& Sherbinin, A. (2014). Resettlement in the twenty-first century. Forced Migration Review, 45, 23-25.

Osmont, A. (2008). A cidade eficaz. Revista Em Pauta, $21,65-80$.
Partridge, W. L. (1989). Involuntary resettlement in development projects. Journal of Refugee Studies, 2(3), 373-384. http://dx.doi.org/10.1093/jrs/2.3.373.

Pereira, I. S. O. (2009). As políticas públicas de revitalização urbana e a localização das classes sociais: o caso de BelémPA (Tese de doutorado em Urbanismo). Programa de Pósgraduação em Urbanismo, Universidade de Brasília, Brasília.

Pereira, J. M. M. (2011). Conflicts and partnerships in the area of socio-environmental projects. Tempo Social, 23(2), 235-263.

Perera, J. (2014). Lose to gain: is involuntary resettlement a development opportunity? Philippines: Asian Development Bank.

Quetulio-Navarra, M., Niehof, A., Vaart, W., Horst, H., \& Se, S. (2012). The disruption and rebuilding of social capital in involuntary resettlement in the Philippines and Indonesia. International Journal of Social Sciences and Humanities Studies, 4(2), 307-323.

Ribeiro, G. B., Fo. (2005). O Banco Mundial e as cidades: construindo instituições na periferia: o caso do PRODUR, Bahia (Tese de doutorado em Planejamento Urbano e Regional). Universidade Federal do Rio de Janeiro, Rio de Janeiro.

Ribeiro, G. B., Fo. (2013). 0 Banco Mundial e as estratégias de desenvolvimento institucional para as cidades: primeiro, ideias, depois dinheiro. In Anais do XI Encontro Nacional da ANPUR (pp. 1-21). Salvador: ANPUR.

Robinson, W. C. (2003). Risks and rights: the causes, consequences, and challenges of development-induced displacement. Washington: Brookings Institution.

Rodrigues, V. (2013). Simbioses de um conflito: desplazamiento e identidade negra na Colômbia. Revista CS, 12(12), 129 156. http://dx.doi.org/10.18046/recs.i12.1679.

Sánchez, F. (2001). A reinvenção das cidades na virada de século: agentes, estratégias e escalas de ação política. Revista de Sociologia e Politica, 16, 31-49.

Santana, J. V. (2012). Desenho ideológico do BID: modelo de gestão no PROMABEN em Belém, Pará. Katálysis, 15(1), 21-31.

Santana, J. V. (2013). Gestão de cidades, infraestrutura e discurso de diminuição da pobreza do BID: notas sobre o Brasil e a Argentina. In M. F. C. M. Gomes (Ed.), Reforma Urbana, mercantilização da cidade e desigualdades socioespaciais (pp. 80-95). Rio de Janeiro: Mauad X. 
Santos, M. (2005). A urbanização brasileira (6. ed.). São Paulo: EdUSP.

Santos, S. (2007). Lamento e dor: uma análise sócioantropológica do deslocamento compulsório provocado pela construção de barragens (Tese de doutorado em Ciências Sociais). Universidade Federal do Pará, Belém.

São Luís. Prefeitura Municipal de São Luís. (2006). Programa de Recuperação Ambiental e Melhoria da Qualidade de Vida da Bacia do Bacanga. Política de Reassentamento Involuntário. São Luís.

São Luís. Prefeitura Municipal de São Luís. (2007). Projeto de aperfeiçoamento da Governança e da qualidade de Vida de São Luís. Melhorias urbanas e gestão ambiental. São Luís. Recuperado em 14 de maio de 2015, de http:// programabaciadobacanga.blogspot. com.br/p/componentes. html

Sá-Silva, J. R., Almeida, C. D., \& Guindani, J. (2009). Pesquisa documental: pistas teóricas e metodológicas. Revista Brasileira de História \& Ciências Sociais, 1(1), 1-15.

Secretaria Municipal Extraordinária de Projetos Especiais - SEMPE. (2015a). Prefeitura Intensifica obras do Programa Bacia do Bacanga no Coroadinho. São Luís. Recuperado em 8 de setembro de 2015, de http://programabaciadobacanga. blogspot.com.br/2015/07/prefeitura-intensifica-obrasdo.html

Secretaria Municipal de Projetos Especiais - SEMPE. (2015b). Prefeitura realiza segunda fase do reassentamento voluntário do Programa Bacia do Bacanga. São Luís. Recuperado em 5 de junho de 2015, de http://programabaciadobacanga. blogspot.com.br/2015/04/prefeitura-realiza-segundafase-do.html

Secretaria Municipal Extraordinária de Projetos Especiais SEMPE. (2015c). Prefeitura de São Luís avança no projeto de urbanização da bacia do Bacanga. São Luís. Recuperado em 20 de maio de 2015, de http://programabaciadobacanga. blogspot.com.br/2015/02/prefeitura-de-sao-luis-avancano.html

Secretaria Municipal Extraordinária de Projetos Especiais - SEMPE. (2015d). Prefeito Edivaldo entrega 496 unidades habitacionais do Residencial Piancó. São Luís. Recuperado em 20 de maio de 2015, de http://programabaciadobacanga. blogspot.com.br/2014/12/prefeito-edivaldo-entrega-496unidades.html

Secretaria Municipal Extraordinária de Projetos Especiais - SEMPE. (2015e). Prefeitura debate com comunidade importância do Canal do Rio das Bicas. São Luís. Recuperado em 20 de maio de 2015, de http://programabaciadobacanga. blogspot.com.br/2014/12/prefeitura-debate-comcomunidade_19.html

Secretaria Municipal Extraordinária de Projetos Especiais - SEMPE. (2015f). Prefeitura e representantes do Banco Mundial vistoriam obras. São Luís. Recuperado em 20 de maio de 2015, de http://programabaciadobacanga. blogspot.com.br/2014/11/prefeitura-e-representantesdo-banco.html

Silva, C. H. L., Jr., Freire, A. T. G., \& Mendes, J. J. (2015). Lógica Fuzzy e Processo Analítico Hierárquico (AHP) na avaliação da qualidade ambiental de nascentes. Revista Eletrônica em Gestão. Educação e Tecnologia Ambiental, 19(2), 292-303.

Silva, G. S., Ferreira, F. G. S., Ferreira, L. C., \& Batalha, M. A. J. (2006). Análise geomorfológica do sistema de drenagem do baixo rio Bacanga: São Luís/MA. In Anais do VI Simpósio Nacional de Geomorfologia. Goiânia: IAG.

Smith, N. (2002). New globalism, new urbanism: gentrification as global urban strategy. Antipode, 34(3), 427-450. http:// dx.doi.org/10.1111/1467-8330.00249.

Sousa, Y. M., Silva de Jesus, L., Santos, J. C. A., Jr., \& Vasconcellos, A. M. A. (2014). Gestão social e governança urbana: o caso do Portal da Amazônia, Belém - PA. Administração Pública e Gestão Social, 6(1), 27-34.

Stewart, D. W., \& Kamins, M. A. (1993). Secondary research: Information sources and methods (2. ed.). Newbury Park: Sage. http://dx.doi.org/10.4135/9781412985802.

Strauss, A., \& Corbin, J. (1998). Basics of qualitative research: Techniques and procedures for developing grounded theory (2. ed.). Thousands Oaks: Sage.

Swyngedouw, E., Moulaert, F., \& Rodriguez, A. (2002). Neoliberal Urbanization in Europe: large-scale urban development projects and the new urban policy. Malden: Blackwell Publishing.

Terminski, B. (2013). Development-induced displacement and resettlement: theoretical frameworks and current challenges. Development, 10, 101.

Trindade, S. C., Jr. (2010). Cidades na floresta: os "grandes objetos" como expressões do meio técnico-científico informacional no espaço amazônico. Revista ICB, 50, 113-137.

Vainer, C. B. (1998). Deslocamentos compulsórios, restriç̃es à livre circulação: elementos para um reconhecimento 
teórico da violência como fator migratório. In Anais do XI Encontro Nacional de Estudos Populacionais. Caxambu: ABEP.

Vainer, C. B. (2003). Utopias urbanas e o desafio democrático. Revista Paranaense de Desenvolvimento, 5, 25-31.

Vandergeest, P. (2003). Land to some tillers: developmentinduced displacement in Laos. International Social Science Journal, 55(175), 47-56. http://dx.doi. org/10.1111/1468-2451.5501005.

Véras, M. P. B. (2010). Cidade, vulnerabilidade e território. Ponto-e-Vírgula: Revista de Ciencias Sociales, 7, 32-48.

Viana, L., \& Fonseca, F. (2006). Impactos sociais e econômicos da atuação do Banco Interamericano de Desenvolvimento nas políticas públicas. Cadernos de Desenvolvimento, 6(9), 199-213.

Vinhote, E. C. A. (2008). Sustentabilidade das formas de ocupação, uso e conservação das matas ciliares nas nascentes do rio Bacanga de São Luís - MA (Dissertação de mestrado em Sustentabilidade de Ecossistemas). Universidade Federal do Maranhão, São Luís.

Wacquant, L. J. D. (2001). Os condenados da cidade: estudos sobre marginalidade avançada. Rio de Janeiro: Revan.

Webber, M., \& McDonald, B. (2004). Involuntary resettlement, production and income: evidence from Xiaolangdi, PRC. World Development, 32(4), 673-690. http://dx.doi.org/10.1016/j. worlddev.2003.10.010.

Wet, C. (2005). Introducing the issues. In C. Wet (Ed.), Development-induced displacement: problems, policies and people (Studies in Forced Migration, Vol. 18, pp. 12-38). New York: Berghahn.

Zuquim, M. L. (2010). Avanços e retrocessos de modelos de intervenção urbanística em assentamentos precários: a experiência de Cubatão, SP. In Anais do I Encontro Nacional ANPARQ. Rio de Janeiro: ANPARQ.

Recebido: Jul. 22, 2015

Aprovado: Dez. 22, 2015 